\title{
Was spricht für die Änderung des Verfassungsartikels?
}

\section{Dorothea Wunder}

PD Dr. med., Fachärztin für Gynäkologie, Centre de Procréation Médicalement Assistée et Endocrinologie Gynécologique, Lausanne

Halbwahrheiten und falsche Annahmen zu diesem komplizierten Thema schüren viele Befürchtungen und Ängste. Doch die Grundsätze Menschenwürde und Respekt vor dem menschlichen Leben bleiben auch bei einer Änderung des Verfassungsartikels erhalten, und das schweizerische Fortpflanzungsmedizingesetz verhindert Missbräuche. Auch in Zukunft würde es keine «unlimitierte Lagerung» von Embryonen geben. Die PID könnte helfen, Abtreibungen zu verhindern.

Ich bin Gynäkologin und seit 1999 im Bereich der Reproduktionsmedizin und gynäkologischen Endokrinologie subspezialisiert. Es mag auf den ersten Blick paradox scheinen, aber obwohl ich für die Verfassungsänderung plädiere, bin ich im Grunde genommen mit den Argumenten der Gegner absolut einverstanden: Menschenwürde und Respekt gegenüber dem menschlichen Leben sind ein Grundprinzip, es muss alles gegen eugenische Tendenzen unternommen werden, usw. Wie ist dieser scheinbare Widerspruch möglich? Der Grund liegt meines Erachtens in Halbwahrheiten und falschen Annahmen zu diesem komplizierten Thema, diese schüren viele Befürchtungen und grosse Ängste.

\section{Worum es geht}

Eine kurze Zusammenfassung: Das Schweizer Volk stimmt am 14.6.2015 darüber ab, ob in Zukunft so viele befruchtete Eizellen in Kultur gesetzt werden können, wie für die optimale Behandlung im individuellen Fall nötig sind. Bisher dürfen maximal 3 befruchtete Eizellen in Kultur gesetzt werden, die restlichen müssen im Vorkernstadium eingefroren werden. Zwischen einer

\section{Es wird auch in Zukunft zu keinem masslosen Einfrieren einer unbegrenzten Anzahl von Embryonen kommen.}

befruchteten Eizelle im Vorkernstadium und einem Embryo im Einzellstadium (ab der Verschmelzung des männlichen und weiblichen Vorkerns handelt es sich definitionsgemäss bereits um einen Embryo) liegen nur wenige Stunden. Die Anzahl der in Kultur gesetzten Eizellen wird sich auch in Zukunft allein schon aus medizinischen Gründen (Gefahr des Überstimula- tionssyndroms bei einer unkontrollierten ovariellen Stimulation) beschränken. Ausserdem wird durch einen neuen Gesetzesartikel die Anzahl der in Kultur gesetzten Eizellen auf 12 beschränkt. Mit anderen Worten: Es wird in Zukunft zu keinem masslosen Einfrieren einer unbegrenzten Anzahl von Embryonen kommen, wie dies viele Gegner glauben. Es ist ausserdem zu beachten, dass es bis heute ebenso wenig zu einem masslosen Einfrieren einer unbegrenzten Anzahl von befruchteten Eizellen im Vorkernstadium gekommen ist, und dies ganz ohne vorgeschriebenen Artikel im schweizerischen Fortpflanzungsmedizingesetz, einfach aus rein medizinischen und ethischen Gründen.

\section{Positive Folgen für In-vitro-Fertilisation und PID}

Der erste grosse Vorteil der Änderung des Verfassungsartikels betrifft alle Paare, die eine In-vitro-Fertilisation-(IVF-)Behandlung machen: denn dadurch, dass Embryonen im Mehrzellstadium eingefroren werden können, wird es möglich sein, durch den Transfer des Embryos mit den besten Chancen einer Implantation die gleichen Schwangerschaftschancen zu erreichen wie mit dem Transfer von 2 Embryonen, die im Vorkernstadium «per Zufall» in Kultur gesetzt und danach transferiert wurden. Damit wird es möglich sein, die Raten an Mehrlingsschwangerschaften nach IVF von heute $15-20 \%$ auf $<5 \%$ zu senken, wie dies in Skandinavien seit 15 Jahren mit Erfolg praktiziert wird. Durch die Reduktion der Mehrlingsschwangerschaften werden Frühgeburtlichkeit, schwere neonatale und maternale Komplikationen, sowie Kosten für das Gesundheitswesen massiv gesenkt. 


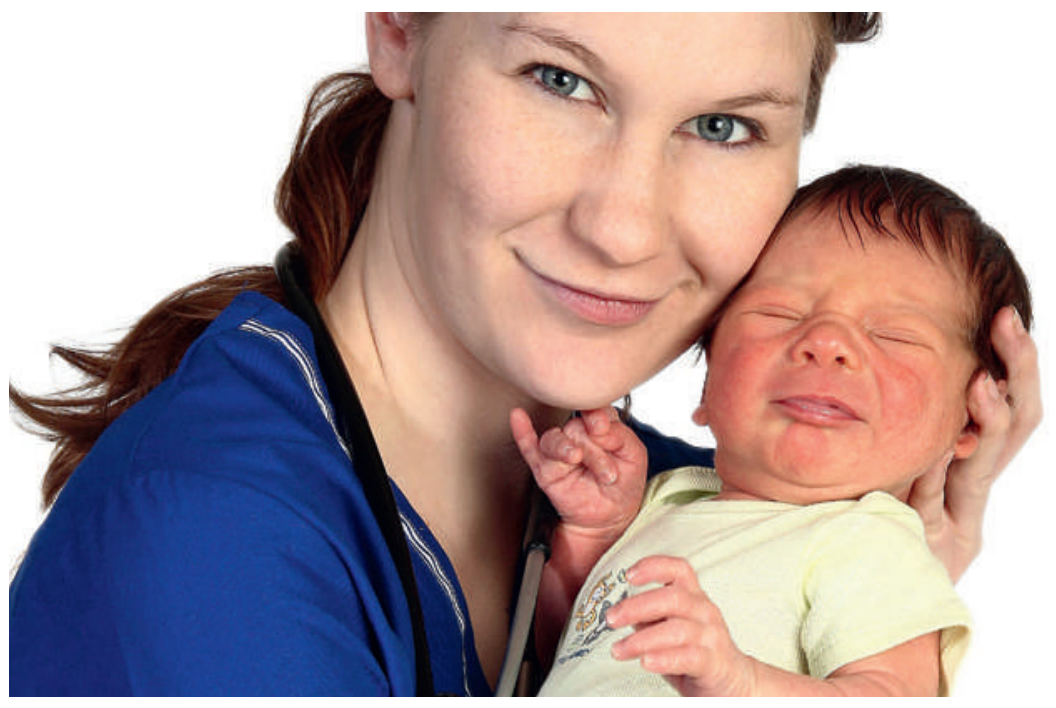

Das grösste Glück: ein gesund geborenes Kind.

Der zweite Vorteil der Änderung der Verfassung ist, dass die Präimplantationsdiagnostik (= PID, die Erkennung schwerer unheilbarer genetischer Erkrankungen im Genom des aus mehreren Zellen bestehenden Embryos vor der Implantation) auch in der Schweiz durchgeführt werden kann. Die PID wurde vor rund 20 Jahren entwickelt und wird seit Jahrzehnten in fast allen europäischen Ländern praktiziert. Es ist festzuhalten, dass die Pränataldiagnostik in der Schweiz erlaubt ist und jede Schwangerschaft bis zum Alter von 3 Monaten (nach Fertigstellung der Organogenese, im Entwicklungsstadium eines Fötus), auch ohne Angabe von Gründen, abgebrochen werden kann.

Damit gibt es durch die Änderung des Verfassungsartikels zwei Vorteile: Unfruchtbare Paare, die eine IVFBehandlung benötigen, haben bessere Chancen, ein gesundes Kind zu bekommen (dies ist durch viele wissenschaftliche Studien bestätigt), und Paare, die Träger einer schweren unheilbaren Erbkrankheit sind, oft schon ein behindertes Kind haben und für die es aus psychischen und ethischen Gründen ausgeschlossen ist, noch ein zweites krankes Kind zu bekommen, können eine Abtreibung nach 12 Wochen Schwangerschaft vermeiden, indem sie die beschwerliche Behandlung einer IVF-Behandlung mit PID machen.

\section{Die Argumente der Gegner}

Schauen wir uns einmal die Argumente der Gegner etwas näher an:

- «Lagerung einer unlimitierten Anzahl von Embryonen, die dann im Labor entsorgt werden»: Ich wäre auch gegen die Lagerung einer unlimitierten Anzahl von Embryonen. Aber wie oben erwähnt, wird es auch in Zukunft keine "unlimitierte Lagerung» von Embryonen geben. Und auch in Zukunft stehen den Paaren alle verschiedenen Möglichkeiten offen (Einfrieren von Embryonen oder von befruchteten Eizellen im Vorkernstadium oder von unbefruchteten Eizellen oder Stimulation von nur 2-3 Eizellen und damit kein Einfrieren), niemand muss Embryonen einfrieren!

- «Ein unbeteiligter Biologe sortiert im Labor die Embryonen und wirft sie nach seinem Gutdünken weg, er entscheidet darüber, was wertes und was unwertes Leben ist." Und: "Ein Tötungsakt, der bei einer Abtreibung allenfalls noch abgewendet werden könnte, geschieht bei der PID anonym. Die mit allen Eigenschaften zum Leben ausgestatteten Embryonen landen im kalten Laborausguss»: Was für eine Horrorvorstellung, ich wäre auch dagegen. Die Realität ist jedoch, dass es medizinisch und gesetzlich klar definierte Indikationen geben wird, wann eine PID durchgeführt wird. Ausserdem wird in stundenlangen Diskussionen vor Beginn einer PID mit den Paaren genauestens besprochen, unter welchen Umständen ein Embryo nicht in die Gebärmutter transferiert wird. Zudem ist zu unterstreichen, dass es sich bei den Paaren um verantwortungsbewusste und schicksalserfahrene Menschen handelt, die am besten über ihre individuelle Situation urteilen können und wissen, ob sie es schaffen, (noch) ein (weiteres) schwerbehindertes Kind grosszuziehen oder nicht. Bei Paaren, die eine PID auf sich nehmen, handelt es sich um Eltern, die es nicht verkraften könnten, ein (weiteres) schwerkrankes Kind aufzuziehen, sie würden sich nach einer pränatalen Diagnose schweren Herzens für eine Abtreibung entscheiden. Das schweizerische Gesetz erlaubt Paaren heute nach der pränatalen Diagnose einer schweren Behinderung des Kindes frei zu entscheiden, eine Abtreibung (theoretisch bis zur 23. Schwangerschaftswoche) durchzuführen, aber niemand ist gezwungen, eine Pränataldiagnostik zu machen. Und genauso wie heute werden Paare auch bei Zulassung der PID in der Zukunft frei entscheiden können, was für sie das Richtige ist: Annehmen eines (weiteren) behinderten Kindes, PID, pränatale Diagnostik, Abtreibung, überhaupt keine genetische Diagnostik und einfach hoffen, usw.

- «Der Embryo kann nur überleben, wenn er eine Prüfung besteht, es handelt sich um eine Konzeption auf Probe»: Ja, die «Konzeption auf Probe» ist seit der Einführung der Pränataldiagnostik und der Möglichkeit der Abtreibung eine Realität, dies hat nichts mit der PID zu tun. Es ist die Entscheidung der zukünftigen Eltern, diese Tests durchzuführen oder nicht und abzutreiben oder nicht. Diese Realität muss akzeptiert werden und wenn vor diesem Hin- 
tergrund klar ist, dass ein individuelles Paar ein Kind mit einer schweren genetischen Erkrankung nicht annehmen kann, muss die Frage gestellt werden: Ist es schlimmer, einen Fötus nach abgeschlossener Organentwicklung abzutreiben, oder zu verhindern, dass es zur Schwangerschaft und danach zur Abtreibung eines kranken Kindes kommt?

- «Behinderte Kinder (und ihre Eltern) werden immer mehr stigmatisiert»: Dies ist eine Fehlvorstellung. Die Akzeptanz von Behinderten in unserer Gesellschaft ist seit Einführung der Pränataldiagnostik nicht gesunken, sondern im Gegenteil gestiegen. Ausserdem hängt die Akzeptanz von behinderten Menschen ja nicht von einer gewissen Anzahl ab. Es gibt überhaupt keinen Hinweis darauf, dass dies nach Einführung der PID in der Schweiz ändern würde, ganz besonders im Hinblick auf die geringe Anzahl der Eltern mit schwerer genetischer Erkrankung und dem geringen Prozentsatz der IVF-Kinder im Allgemeinen (es würde selbst im Falle eines Aneuploidie-Screenings, das heute auch im Ausland weder routinemässig durchgeführt, noch State of the Art ist, pro Jahr maximal ein Embryo mit Trisomie 21 diagnostiziert).

- «Glück oder Leid kann nicht diagnostiziert werden»: Absolut richtig. Ausserdem kann eine genetische Diagnostik weder alle Behinderungen noch Malformationen ausschliessen, ganz abgesehen von den möglichen Schwangerschaftskomplikationen mit Frühgeburtlichkeit, neonataler Morbidität usw. Es gibt keine Sicherheit im Leben, weder mit noch ohne genetische Diagnostik, und es wird immer Behinderte geben und Menschen mit schweren genetischen Erkrankungen, Malformationen, schweren physischen und psychischen Erkrankungen usw. Aber kann diese Tatsache als Argument verwendet werden, Paaren mit einer schweren genetischen Erkrankung, die ihrem Kind und ihrer Familie Leid ersparen wollen, eine PID vorzuenthalten?

- "Das Aneuploidie-Screening ist ein totales genetisches Screening und wird bei allen Embryonen angewendet werden»: Welch Horrovorstellung, dass in Zukunft jeder Embryo genetisch komplett durchgescreent und je nach Resultat weggeworfen wird. Dem ist nicht so, auch in Europa nicht, die Technik ist sehr heikel und nicht State of the Art. Ausserdem handelt es sich beim Aneuploidie-Screening auch nicht um ein «komplettes genetisches Screening», sondern es werden die gleichen chromosomalen Anomalien gesucht wie bei der Pränataldiagnostik. Das Aneuploidie-Screening wäre nur in Spezialfällen indiziert, zum Beispiel bei einer individuellen Sterilitätspatientin, die zur Erfüllung ihres Kinderwunsches eine IVF-Behandlung machen muss und gleichzeitig ein erhöhtes Risiko für ein Kind mit Trisomie 21 hat, jedoch ein Kind mit einer Trisomie 21 abtreiben würde. Durch das Vermeiden des Transfers eines Embryos mit Trisomie 21 könnte man dieser Patientin einen Schwangerschaftsabbruch ersparen.

- «Die Präimplantationsdiagnostik ist gefährlich und stellt ein Risiko für Eugenik dar»: Man kann die Gefahr eines zukünftigen Missbrauchs weltweit nie zu 100 Prozent ausschliessen. Aber kann die hypothetische Annahme eines Missbrauchs irgendwo in der Welt ein Argument dafür sein, diese Technik, die für gewisse Paare ein Segen ist und die ausserdem in der Schweiz durch unser Fortpflanzungsmedizingesetz sorgfältig definierte, strenge und ethisch akzeptierte Indikationen hat, zu verbieten? Die Weltgeschichte hat gezeigt, dass das Risiko eugenischen Denkens seit langem existiert. Es hat seine Ursache nicht in (neuen) genetischen Techniken, sondern in ideologisch falsch geprägtem Denken und ist vornehmlich in totalitären Regimen zu finden. Es ist Aufgabe des Staates, eugenischen Tendenzen, sollten sie aufkommen, Einhalt zu gebieten. Aber diese hypothetischen Befürchtungen können doch nicht als Argument verwendet werden, Paaren die Möglichkeit dieser medizinischen Behandlung zu verbieten. Ganz besonders wenn man bedenkt, dass das IVF-Verfahren für die betroffene Frau eine lange, physisch und psychisch stressige, invasive und schwere Behandlung darstellt und der Erfolg (= die Geburt eines gesunden Kindes) alles andere als garantiert ist. Ganz abgesehen davon bestehen theoretisch die gleichen Risiken der «Eugenik» mit der in der Schweiz seit langem praktizierten Pränataldiagnostik, die nach Spontankonzeption und ohne mühsame, schwere und teure IVF-Behandlung durchgeführt werden kann.

\section{Fazit}

Zusammengefasst kann gesagt werden, dass die Grundsätze Menschenwürde und Respekt vor dem menschlichen Leben mit der Änderung des Verfassungsartikels erhalten bleiben und der Rahmen des schweizerischen Fortpflanzungsmedizingesetzes Missbräuche verhindert. Dank der Verfassungsänderung können in Zukunft Mehrlingsschwangerschaften nach IVF von 20\% heute auf $<5 \%$ reduziert und damit das Risiko schwerer neonataler und maternaler Komplikationen sowie die Kosten im Gesundheitswesen beträchtlich gesenkt werden. Ausserdem kann die PID in Zukunft Abtreibungen bei Paaren mit einer schweren genetischen Erkrankung verhindern. 